\title{
Revista Colombiana de

\section{Relevancia clínica de la interacción de la warfarina y del acetaminofén: estudio de cohortes retrospectivo}

\author{
Mauricio Ceballos ${ }^{a, *}$, César A. González ${ }^{b}$, Héctor A. Holguín ${ }^{c}$ \\ y Pedro Amariles ${ }^{c}$
}

\begin{abstract}
a Grupo de Investigación Promoción y Prevención Farmacéutica, Grupo de Investigación Tecnología en Regencia de Farmacia, Facultad de Ciencias Farmacéuticas y Alimentarias, Universidad de Antioquia UdeA, Medellín, Colombia

b Departamento de Farmacia, Facultad de Ciencias Farmacéuticas y Alimentarias, Universidad de Antioquia, Medellín, Colombia

c Grupo de Investigación Promoción y Prevención Farmacéutica. Facultad de Ciencias Farmacéuticas y Alimentarias,

Universidad de Antioquia, Medellín, Colombia
\end{abstract}

Recibido el 3 de julio de 2015; aceptado el 27 de octubre de 2015

Disponible en Internet el 23 de diciembre de 2015

\section{PALABRAS CLAVE \\ Tratamiento anticoagulante; Factores de riesgo; Hemorragia}

\begin{abstract}
Resumen
Objetivo: Evaluar el uso concomitante de la warfarina y el acetaminofén en una población ambulatoria de pacientes anticoagulados, como posible factor de riesgo en el aumento de la Relación Normalizada Internacional (INR).

Métodos: Estudio de cohortes retrospectivo. Se analizó la información de 1.458 pacientes anticoagulados con la warfarina. El factor de riesgo fue la utilización conjunta de warfarinaacetaminofén; el tiempo de seguimiento fue de 1 mes y como evento final se consideró un incremento del INR igual o mayor a 0,5 unidades. La asociación del factor de riesgo con el evento final se estableció con el riesgo relativo (RR) y el riesgo atribuible poblacional (RAP).

Resultados: 63 pacientes cumplieron criterios de inclusión, 21 pacientes expuestos y 42 pacientes no expuestos. En los individuos expuestos se observó un aumento estadísticamente significativo en el INR al mes siguiente de haber iniciado el tratamiento con el acetaminofén comparado con el momento de ingreso al estudio (mes 1: mediana 3,06 vs. mes 0: mediana 2,63 ), (valor $p=0,003$ ). En contraste, en los sujetos no expuestos no se observaron diferencias estadísticamente significativas en los valores del INR (mes 0: mediana 2,63 vs. mes 1: mediana $2,75)$, (valor $p=0,115$ ). El uso de la warfarina y el acetaminofén representó un RR de 2,5 veces mayor de incrementar el INR mayor o igual de 0,5 unidades.
\end{abstract}

\footnotetext{
* Autor para correspondencia.

Correo electrónico: javier.ceballos@udea.edu.co (M. Ceballos).
} 
Conclusión: El uso concomitante de la warfarina y el acetaminofén está asociado a un aumento del INR igual o mayor de 0,5 unidades, lo cual podría generar un mayor efecto anticoagulante $y$, por tanto, un mayor riesgo potencial de sangrado.

(C) 2015 Sociedad Colombiana de Cardiología y Cirugía Cardiovascular. Publicado por Elsevier España, S.L.U. Este es un artículo Open Access bajo la licencia CC BY-NC-ND (http:// creativecommons.org/licenses/by-nc-nd/4.0/).

\section{KEYWORDS \\ Blood-thinning \\ treatment; \\ Risk factors; \\ Haemorrage}

\section{Clinical relevance of the interaction of warfarin and acetaminophen: retrospective cohort study}

\begin{abstract}
Motivation: To assess concomitant use of warfarin and acetaminophen in an outpatient population of patients receiving blood-thinning drugs as a possible risk factor for the increase of International Normalised Ratio (INR).

Methods: Retrospective cohort study. The information pertaining 1,458 patients receiving blood-thinning drugs with warfarin was analysed. The risk factor was the joint intake of warfarin and acetaminophen; follow-up time was 1 month and the final event considered was a INR increase of or higher than 0.5 units. The association of the risk factor with the final event was establish with the Relative Risk (RR) and the Population Attributable Risk (PAR).

Results: 63 patients met the inclusion criteria, of whom 21 were exposed patients and 42 were unexposed patients. In the exposed patients a statistically significant increase of INR on the month following the onset of treatment with acetaminophen was observed, compared to the baseline values (month 1: median 3.06 vs. month 0 : median 2.63$),(p=0.003)$. In contrast, no statistically significant differences of INR values were observed in unexposed individuals (month 0 : median 2.63 vs. month 1 : median 2.75$)(p=0.115)$. The combination of warfarin and acetaminophen represented a RR 2.5 times higher to increase INR greater than or equal to 0.5 units.

Conclusion: Concomitant use of warfarin and acetaminophen is associated to an increase of INR equal to or greater than 0.5 units, which could generate a higher blood-thinning effect and, thus, cause a potentially higher risk of bleeding.

(C) 2015 Sociedad Colombiana de Cardiología y Cirugía Cardiovascular. Published by Elsevier España, S.L.U. This is an open access article under the CC BY-NC-ND license (http:// creativecommons.org/licenses/by-nc-nd/4.0/).
\end{abstract}

\section{Introducción}

La warfarina es utilizada para reducir los problemas tromboembólicos en pacientes con Fibrilación Atrial (FA), Trombosis Venosa Profunda (TVP), Tromboembolismo Pulmonar (TEP) o Prótesis Valvular (PV) ${ }^{1}$. Su eficacia y seguridad se ven afectadas por: su alta variabilidad farmacocinética y farmacodinámica ${ }^{2,3}$, la mutación de la isoenzima CYP2C $9^{4}$, el uso en pacientes de edad avanzada $^{5}$ y las numerosas interacciones medicamentosas ${ }^{6}$; desencadenando principalmente, eventos hemorrágicos ${ }^{7-10}$.

Es frecuente el uso concomitante de la warfarina y los antiinflamatorios no esteroideos (AINEs), los cuales pueden tener interacción con la warfarina ${ }^{11}$. Para el acetaminofén, diferentes estudios muestran que existe una estrecha relación temporal y dosis dependiente en el incremento del INR $^{3,12-14}$, a dosis del acetaminofén de dos gramos al día ${ }^{15}$.

La interacción medicamentosa se presenta debido al bloqueo de las enzimas del ciclo de la vitamina $\mathrm{K}$ por el metabolito $\mathrm{N}$-acetil-parabenzoquinona-imina (NAQPI) del acetaminofén ${ }^{16-18}$. Una revisión estructurada clasificó la relevancia clínica de la interacción medicamentosa como riesgo alto (nivel 2$)^{19,20}$. La literatura recomienda utilizar una dosis del acetaminofén menor a $2 \mathrm{~g}$ al día por 3-4 días como máximo para disminuir el riesgo de sangrado $3,13,15,21$.

Existe controversia sobre la evidencia disponible que respalde el efecto y la relevancia clínica de dicha interacción, especialmente, por la ausencia de información de estudios realizados en Colombia. Por tanto, el objetivo de este estudio fue evaluar el uso concomitante de la warfarina y el acetaminofén en una población ambulatoria de los pacientes anticoagulados, como posible factor de riesgo (FR) en el aumento del INR.

\section{Métodos}

Estudio observacional de cohortes retrospectivo, a partir de una población del programa de pacientes anticoagulados con la warfarina de una aseguradora de la ciudad de Medellín, Colombia, valorada entre enero del año 2008 y diciembre del año 2009. El tiempo de seguimiento fue de 30 días, con el fin de obtener dos mediciones del INR por cada paciente (al inicio y al final del seguimiento).

Se determinó un tamaño de la muestra de 36 pacientes (12 expuestos y 24 no expuestos), teniendo en cuenta los siguientes indicadores muéstrales: una incidencia del $94 \%$ 
(aumento del INR $\geq 0,5$ unidades) en los pacientes expuestos al acetaminofén y una incidencia del $28 \%$ en aquellos individuos no expuestos al acetaminofén, según lo reportado por Mahe, et $\mathrm{al}^{12}$. Debido a que el tamaño de muestra calculado fue muy pequeño, se tomó como la muestra de pacientes expuestos y no expuestos; todos los pacientes que cumplieron los criterios de inclusión y exclusión evaluados en el programa de pacientes anticoagulados con la warfarina durante los 24 meses escogidos, para la selección de la muestra (enero del año 2008 - diciembre del año 2009). Como factor de riesgo se consideró la utilización conjunta del acetaminofén y la warfarina. En el caso del acetaminofén, a dosis terapéuticas según su indicación como analgésico o como antipirético $(0,5$ a $4 \mathrm{~g} /$ día), y para el caso de la warfarina se tuvieron en cuenta las dosis terapéuticas acorde a su valor de INR.

Se incluyeron pacientes mayores de 18 años de edad, en tratamiento con la warfarina mínimo 6 meses previos y que fueran considerados como pacientes controlados según los siguientes criterios: a) su medición del INR y la medición anterior a esta (mes 0 y mes -1 , respectivamente), se encontraban en los rangos terapéuticos, acorde con su patología de base $(2,5 \pm 0,5$ para la patología no valvular $y$ $3,5 \pm 0,5$ para la protesis valvular); y b) las diferencias entre las dos mediciones fuera menor del $15 \%$, acorde con Choi, et $\mathrm{al}^{22}$. Se excluyeron los pacientes con modificaciones en: la dosis de la warfarina por cualquier justificación clínica entre el mes -1 y el mes 0 , la historia de consultas por urgencia $u$ hospitalización en los últimos 6 meses previos al estudio, los problemas de adherencia al programa de anticoagulados en los últimos 6 meses, la hipoalbuminemia o las enfermedades asociadas a disminución de albumina plasmática, la historia de alcoholismo o tabaquismo, el cáncer, la prescripción de antibióticos, la diarrea aguda, los cambios en la posología o en la farmacoterapia y la automedicación del acetaminofén.

Fueron considerados como cohorte expuesta aquellos pacientes controlados en su patología trombótica y que, al momento de ingresar al estudio (mes 0), el médico tratante les prescribió acetaminofén (FR). Como cohorte no expuesta se consideró a los pacientes controlados en su patología trombótica sin prescripción del acetaminofén, al momento de ingresar al estudio (mes 0 ).

Para controlar el FR, se descartaron los pacientes que utilizaron el acetaminofén el mes anterior (mes -1) al mes de ingreso al estudio (mes 0). Adicionalmente, se controló que a todos los pacientes les suministraron la warfarina del mismo laboratorio fabricante, buscando disminuir variaciones clínicamente relevantes en el INR ${ }^{23-26}$.

El paciente adherente fue aquel que cumplió el $100 \%$ de las consultas mensuales de seguimiento en el programa de los anticoagulados y acudió por sus medicamentos durante los últimos seis meses antes de ingresar al estudio. En todos los casos se verificó la ausencia de cambios en los medicamentos prescritos y utilizados desde el mes anterior.

La información se tabuló y procesó en el software STATA ${ }^{\circledR}$ versión 11 para los análisis estadísticos con una confianza del $95 \%$. A las variables cuantitativas se les calculó la mediana y por su tamaño de muestra pequeño y a las variables cualitativas se les calcularon las frecuencias absolutas y relativas.

Para determinar el cambio en el INR, debido a la interacción medicamentosa entre la warfarina y el acetaminofén, se realizó un análisis bivariado. Los análisis bivariados en las variables cualitativas se realizaron a través de la Prueba Chi-cuadrado o test exacto de Fisher cuando las frecuencias esperadas fueron menores de 5 en el $25 \%$ o más de las frecuencias observadas. En las variables cuantitativas se utilizó el test $U$ de Mann-Whitney en las comparaciones entre los expuestos y los no expuestos, y para las comparaciones de las variables con el evento se utilizó la prueba de los rangos con signo de Wilcoxon (INR igual o mayor a 0,5).

Con el fin de controlar sesgos de confusión, se realizó una estratificación en variables como la edad ( $\geq 70$ años), el género, en aquellas variables que los investigadores consideraron clínicamente relevantes o que en el análisis univariado tuvieron diferencias significativas. Se realizaron estratificaciones con diferentes variables dicotómicas o que se dicotomizaron para ello, teniendo como estadístico de prueba el test de Manthel y Haenszel. Para identificar otros factores sociodemográficos y/o clínicos que pudieran incrementar el INR, se realizó un análisis de Regresión Logística Multivariante, teniendo en cuenta las variables "candidatas" a incluirse en el modelo posterior, seleccionadas de acuerdo con su significancia $(p<0.25$ según criterio de Hosmer-Lemeshow).

Para determinar la asociación que podrían tener las variables sociodemográficas y clínicas con un aumento del INR igual o mayor a 0,5 unidades, se procedió inicialmente a explorar su relación mediante el análisis bivariado, incluyendo en el modelo las variables que mostraron una significancia ( $p<0,25$ según criterio de Hosmer-Lemeshow). Finalmente, se calcularon las medidas de asociación entre el FR y el incremento del INR igual o mayor a 0,5 unidades, a través del cálculo del riesgo relativo (RR), así como el cálculo del riesgo atribuible poblacional (RAP), en ambos casos, teniendo en cuenta sus intervalos de confianza al $95 \%$.

\section{Consideraciones éticas}

El estudio se ajustó a los lineamientos de la Resolución 8430 de 1993 del Ministerio de Salud ${ }^{27}$. La investigación se consideró sin riesgo. El uso de la historia clínica se realizó previa autorización de la institución. El estudio fue aprobado por el comité de ética de investigación de la institución y por el comité operativo de investigación de la Facultad de Medicina de la Universidad CES.

\section{Resultados}

Se revisaron los registros de dispensación y las historias clínicas electrónicas de 1.458 pacientes en terapia anticoagulante con la warfarina y que hacían parte del programa de pacientes anticoagulados ambulatorios entre enero del año 2008 y diciembre del año 2009; 63 de estos pacientes cumplieron los criterios de inclusión y exclusión, de los cuales, $21(33,3 \%)$ pacientes se clasificaron como expuestos al FR y 42 pacientes $(66,6 \%)$, como no expuestos al FR. En la figura 1 se presenta el flujo de pacientes en el estudio.

Las características sociodemográficas y clínicas más relevantes se observan en la tabla 1. De los 63 pacientes incluidos, $37(58,7 \%)$ eran hombres y $31(49,2 \%)$ tenían una edad mayor o igual a 70 años. De los 63 pacientes, el $77,8 \%$ presentaban FA y PV $(41,3$ y $36,5 \%$, respectivamente) y $22,2 \%$ recibieron la warfarina para prevenir recidivas de 


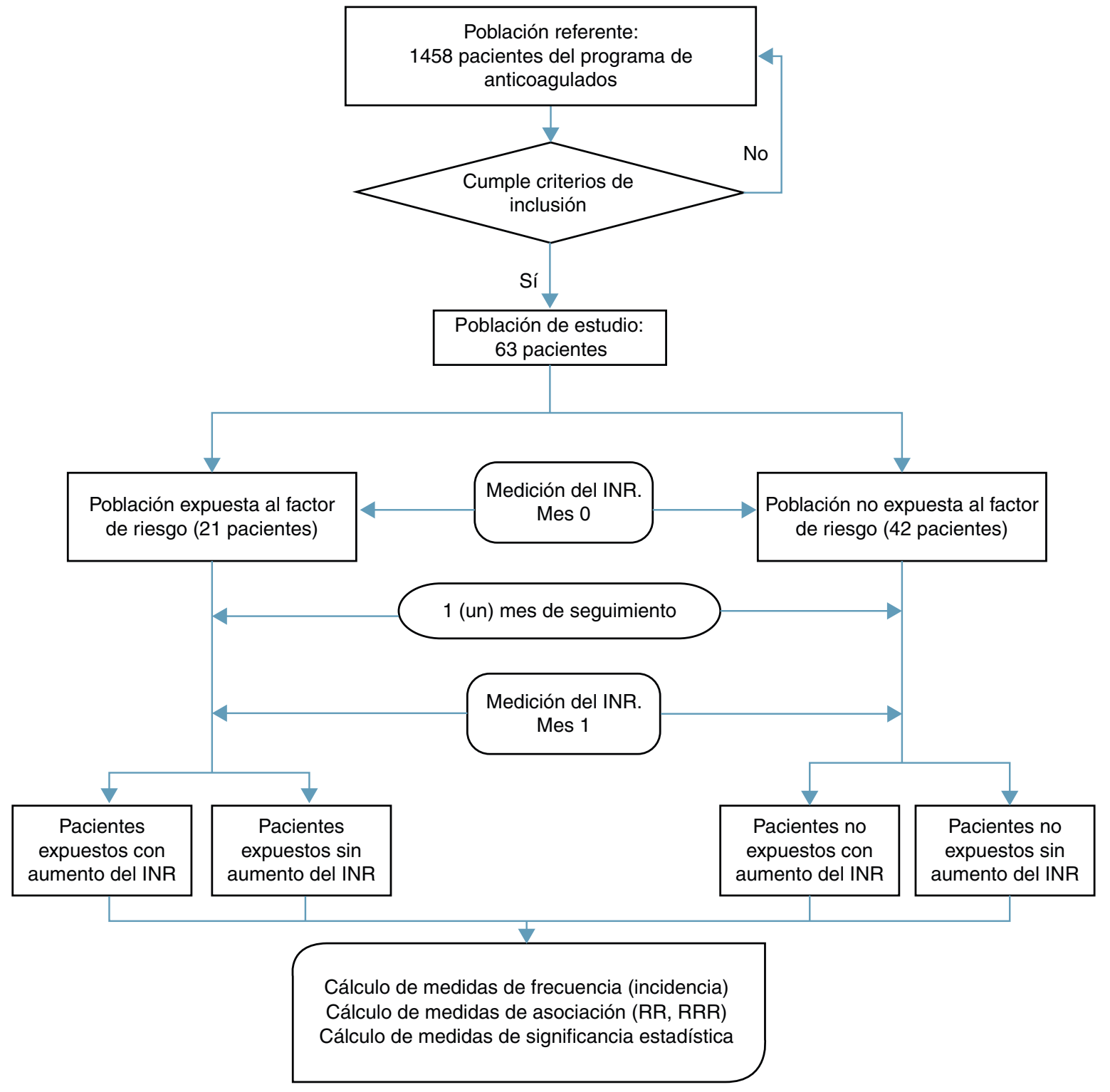

Figura 1 Características metodológicas y flujo de pacientes en el estudio.

TVP y TEP (9,5 y 12,7\%, respectivamente). El 33,3\% de los pacientes tenían dos o más FR cardiovasculares como: hipertensión arterial, diabetes mellitus u obesidad. Al momento de ingreso al estudio la dosis mediana de la warfarina fue de $32,5 \mathrm{mg} / \mathrm{semana}$ (RI: $25-42,5$ ). No se observaron diferencias significativas en la dosis de la warfarina entre los grupos estudiados (mediana grupo expuesto: $30 \mathrm{mg} / \mathrm{semana}$ (RI: 26,2-36,3), versus mediana no expuestos: $35 \mathrm{mg} / \mathrm{semana}$ (RI: 24,3-45), $p=0,569$ ).

Las enfermedades de riesgo cardiovascular se presentaron en el $77 \%$ de los pacientes (mediana: 2 ). No se observaron diferencias estadísticamente significativas en la cantidad de los FR cardiovascular entre los grupos estudiados ( $p=0,137)$. Adicionalmente, las proporción de pacientes polimedicados en el grupo de expuestos fue similar a la observada en el grupo de no expuestos ( $38,1 \%$ vs. $57,1 \% ; p=0,154)$.
La proporción de pacientes que utilizaron medicamentos inhibidores enzimáticos del metabolismo hepático de la warfarina fue de $23,8 \%$. Se encontraron diferencias significativas entre ambos grupos con los siguientes inhibidores: la lovastatina $28,6 \%$ en los expuestos y $66,7 \%$ en los no expuestos $(p=0,007)$ y con la amiodarona, $14,3 \%$ pacientes en los no expuestos y ninguno en los expuestos $(p=0,033)$.

De los 21 pacientes expuestos al FR, 11 (52\%), utilizaron semanalmente $10,5 \mathrm{~g}$ o más del acetaminofén (RI: 5,2-14), la dosis semanal osciló entre los 3,5 a 28,0 g; y además, se les administró el acetaminofén durante 10 días o más (RI: 1030). La analgesia, fue la principal indicación en los pacientes expuestos para la cual se utilizó el acetaminofén (90,5\%). Del total de pacientes expuestos al FR, a $19(90,4 \%)$, se les prescribió 2 gramos/día o menos de acetaminofén. 
Tabla 1 Características sociodemográficas y clínicas

\begin{tabular}{|c|c|c|c|}
\hline & Expuestos $n=21$ & $\begin{array}{l}\text { No expuestos } \\
n=42\end{array}$ & Valor $\mathrm{p}$ \\
\hline Edad en años (media, DE) & $65,2(16,1)$ & $66,8(12,1)$ & 0,988 \\
\hline Género masculino (n, \%) & $6(28,7)$ & $31(73,8)$ & 0,001 \\
\hline IMC $\left(\mathrm{kg} / \mathrm{m}^{2}\right)$ (media, $\left.D E\right)$ & $27,8(5,9)$ & $26,3(3,8)$ & 0,804 \\
\hline \multicolumn{4}{|l|}{ Indicación Warfarina ((n, (\%)) } \\
\hline Trombosis venosas profunda & $5(7,9)$ & $3(4,8)$ & 0,104 \\
\hline Fibrilación auricular & $8(12,7)$ & $18(28,6)$ & 0,717 \\
\hline Trombosis pulmonar & $2(3,2)$ & $4(6,3)$ & 1,000 \\
\hline Prótesis valvular & $6(9,5)$ & $17(27,0)$ & 0,355 \\
\hline \multicolumn{4}{|l|}{ Otros problemas de salud (n, (\%)) } \\
\hline Trastornos del sistema nervioso & $2(2,5)$ & $5(11,9)$ & 1,000 \\
\hline Trastornos endocrinos & $5(23,8)$ & $7(16,7)$ & 0,513 \\
\hline Factores de riesgo cardiovascular & $15(71,4)$ & $34(81,0)$ & 0,522 \\
\hline Hipertensión & $5(23,8)$ & $24(57,1)$ & 0,016 \\
\hline Diabetes Mellitus & $3(14,3)$ & $8(19,0)$ & 0,738 \\
\hline Dislipidemia & $9(42,9)$ & $21(50,0)$ & 0,593 \\
\hline Dosis media de la warfarina ( $\mathrm{mg} /$ semana) (DE) & $32,6(8,08)$ & $35,7(16,11)$ & 0,569 \\
\hline \multicolumn{4}{|l|}{ Número de pacientes con: $(n,(\%))$} \\
\hline INR incremento & $5(7,9)$ & $3(4,8)$ & 0,074 \\
\hline INR incremento $>0,5$ & $9(14,3)$ & $7(11,1)$ & 0,028 \\
\hline IMC > 1INR incremento de 0,1 a 0,49 & $15(23,8)$ & $20(31,7)$ & 0,063 \\
\hline
\end{tabular}

INR: Relación Normalizada Internacional; IMC: Índice de Masa Corporal.

\section{Efecto del acetaminofén en el aumento del INR mayor o igual a 0.5 unidades}

Como se observa en la fig. 2 , en el grupo de expuestos se presentó un aumento estadísticamente significativo en el INR mayor o igual a 0.5 unidades (mes 1: mediana 3,06; RI: 2,463,61 vs. mes 0 : mediana: 2,60; RI: 2,25-2,81), $(p=0,003)$. En contraste, en el grupo de pacientes no expuestos no se observaron diferencias en los valores del INR entre los tiempos evaluados (mes 0: mediana: 2,63; RI: 2,20-3,12 vs. mes 1: mediana: 2,75; RI: 2,38-3,21; $\mathrm{p}=0.115)$.

\section{Riesgo de incremento del INR igual o mayor a 0,5 unidades con el uso conjunto de la warfarina y el acetaminofén}

En los pacientes que recibieron la warfarina conjuntamente con el acetaminofén, el riesgo de incrementar el INR $>0,5$ entre el mes 0 y el mes 1 fue 2,5 veces mayor que en los pacientes que no utilizaron el acetaminofén (RR: 2,5; IC: 1,11-5,94; valor $p=0,027)$. El 61\% del incremento del INR igual o mayor a 0,5 unidades que se presentó en este grupo poblacional se debe al uso concomitante de la warfarina y el acetaminofén, por tanto, el control de este FR podría disminuir la incidencia del desenlace final.

\section{Otros factores asociados con el aumento del INR igual o mayor a 0,5}

Las variables incluidas en el análisis bivariado fueron: HTA $(p=0,036)$, edad igual o mayor a 70 años $(p=0,094)$, dosis de la warfarina igual o mayor a $50 \mathrm{mg} / \mathrm{semana}(\mathrm{p}=0,156)$, INR inicial (mes 0$)$ entre 2,5-3, ( $p=0,170)$ e INR inicial (mes 0 ) mayor de $3(p=0,131)$. Los resultados del análisis bivariado se resumen en la tabla 2.

En la tabla 3, se presenta el análisis estratificado de las variables que pudieran ser factores de confusión en el incremento del INR mayor o igual a 0,5 unidades. Para el análisis multivariado todas las variables independientes fueron ingresadas utilizando el método "Enter"'. Posteriormente, con la ayuda del software estadístico se utilizaron los métodos automáticos "Forward" y "Backward", y se procedió a realizar una regresión "paso a paso"'. El modelo de regresión logística multivariante final estuvo conformado por cinco variables independientes recodificadas: edad igual o mayor a 70 años, dosis de la warfarina igual o mayor $50 \mathrm{mg} /$ semana, exposición al FR, HTA, INR inicial (mes 0) mayor o igual a 3 . Observando que las variables dosis de la warfarina igual o mayor a $50 \mathrm{mg} / \mathrm{semana}$ y la edad igual o mayor a 70 años fueron estadísticamente significativas, concluyendo que ambas variables aumentan el riesgo de incremento del INR igual o mayor a 0,5 unidades, Los resultados del análisis multivariado se muestran en la tabla 4.

\section{Eventos clínicos significativos en pacientes con la warfarina}

No se presentaron reacciones adversas, muertes, eventos de sangrado ni hospitalizaciones en ninguno de los dos grupos expuestos y no expuestos durante el tiempo de seguimiento. 


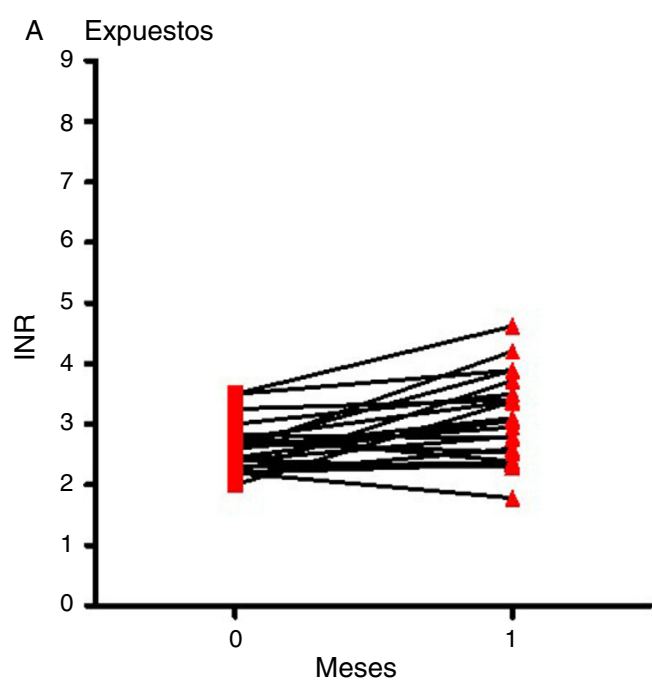

Wilcoxon signed rank test $P$ value
B No expuestos

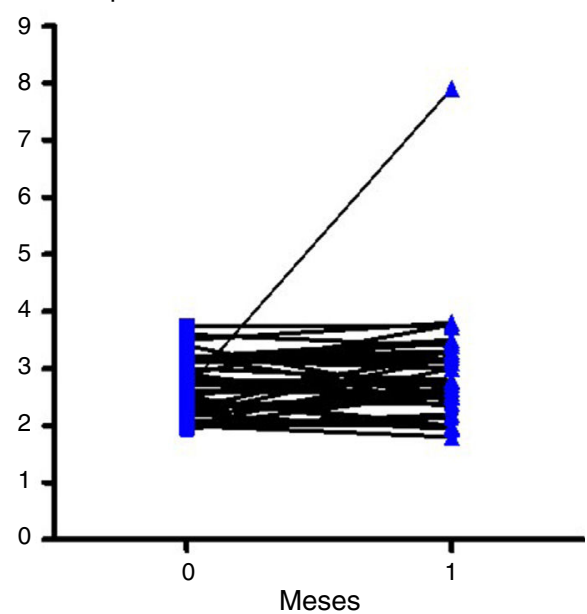

Wilcoxon signed rank test $P$ value

Figura 2 Comparación entre las mediciones del INR.

\section{Discusión}

En este estudio se evidenció que $1.456(66,3 \%)$ de los pacientes que fueron valorados en el programa de anticuagulados entre enero del año 2008 y diciembre del año 2009, se les prescribió por lo menos una vez el acetaminofén. Resultados similares a los reportados por Kotirum et al., ${ }^{28}$, quienes reportaron una frecuencia de prescripción del acetaminofén conjuntamente con la warfarina del 63,15\% $(n=1.093)$. Por su parte, muy superior al reportado en el Reino Unido ${ }^{29}$ en el cual se encontró una frecuencia de consumo solo del $37,26 \%$ $(n=4.152)$.

En este estudio se utilizó como evento o desenlace final el incremento del INR igual o mayor a 0,5 unidades, soportado por dos ECC realizados por Mahe en los años 2005 y 2006 12,13. En estos ECC se comparó la utilización de 4 gramos/día del acetaminofén versus placebo en pacientes anticoagulados con la warfarina. En ambos estudios se evidenció que un paciente anticoagulado con la warfarina, la exposición a 4 gramos/día del acetaminofén se asoció con un incremento del INR de 1,04 $\pm 0,55$ en el primer estudio y de $1,20 \pm 0,62$ en el segundo estudio. Basados en los resultados de dichos estudios se puede inferir que el mínimo aumento en el INR fue aproximadamente, igual o mayor de 0,5 unidades.

El presente estudio encontró que, en 9 (42,9\%) de pacientes anticoagulados con la warfarina y que utilizaron concomitantemente el acetaminofén (21 expuestos), se presentó un aumento del INR igual o mayor a 0,5 unidades. Dado que la dosis promedio de la warfarina en los pacientes expuestos y no expuestos, no presentó diferencias estadísticamente significativas, se puede inferir que dicho incremento del INR no está asociado a la cantidad de la warfarina que utilizó el paciente. Además, durante el período de recolección de datos de este estudio (24 meses) se dispensó siempre la misma marca de la warfarina, controlando con ello la variabilidad en el INR que pudo estar asociada a una marca específica, como lo reportaron Builes, et al. $(2010)^{23}$, en cuyo estudio, llevado a cabo en Colombia, demostraron dificultades en el manejo del INR y que la frecuencia de episodios de sangrado menor es significativamente mayor entre los pacientes anticoagulados con la warfarina genérica que en los anticoagulados con el Coumadin ${ }^{\circledR}$. No obstante, otros estudios publicados a nivel mundial han demostrado que no existen diferencias en el INR cuando se hace el cambio de una marca de la warfarina a otra ${ }^{24-26}$.

Al igual que otros estudios previamente publicados $3,12,13,15$, en los cuales se encontró asociación entre el uso concomitante de la warfarina y el acetaminofén y el aumento significativo del INR, el presente estudio encontró que existe 2,5 veces más riesgo (RR) de aumentar el INR igual o mayor a 0,5 unidades, cuando el paciente utiliza la warfarina y el acetaminofén que aquel paciente que no utiliza dicha combinación de medicamentos. No obstante, un trabajo no respalda la alteración en la respuesta por el uso del acetaminofén en la terapia anticoagulante ${ }^{14}$, lo que puede deberse a la metodología utilizada en ese trabajo y las dosis de los fármacos no son comparables en la práctica clínica.

Al correlacionar algunas características demográficas y clínicas del presente estudio con el aumento del INR igual o mayor a 0,5 unidades, no se observaron diferencias significativas en ninguna de ellas. Resultados similares fueron reportados por Hylek, et $\mathrm{al}^{3}$, y Choi, et $\mathrm{al}^{22}$, en los cuales las variables sociodemográficas fueron controladas desde la selección de los pacientes. Choi, et $\mathrm{al}^{22}$, encontraron, además, de los factores descritos, que: la edad, el género, el IMC y el INR basal se asociaron con aumentos del INR en más del $15 \%$. En este estudio, el análisis de dichas variables y otras más, a través de análisis bivariados y estratificado, 
Tabla 2 Comparación de otros factores que pudieran aumentar el INR igual o mayor a 0,5 unidades en los pacientes anticoagulados con la warfarina

\begin{tabular}{|c|c|c|c|c|c|}
\hline $\begin{array}{l}\text { Variables } \\
\text { cualitativas }\end{array}$ & INR $\geq 0,5$ & INR $<0,5$ & Valor $\mathrm{p}^{\mathrm{a}, \mathrm{b}}$ & RR & IC del RR \\
\hline & n (\%) & n (\%) & & & \\
\hline \multicolumn{6}{|l|}{ Género } \\
\hline Masculino & $9(24,3)$ & $28(75,7)$ & 1,000 & 0,903 & $0,386-2,116$ \\
\hline \multicolumn{6}{|c|}{ Enfermedades concomitantes } \\
\hline Endocrinas & $3(25)$ & $9(75)$ & 1,000 & 0,981 & $0,331-2,907$ \\
\hline Sistema nervioso & $0(0)$ & $7(100)$ & - & - & - \\
\hline $\begin{array}{l}\text { Riesgo } \\
\text { cardiovascular }\end{array}$ & $11(22,4)$ & $38(77,6)$ & 0,315 & 0,629 & $0,262-1,507$ \\
\hline Dislipidemia & $7(23,3)$ & $23(76,7)$ & 0,778 & 0,856 & $0,364-2,012$ \\
\hline Diabetes & $2(18,2)$ & $9(81,8)$ & 0,714 & 0,675 & $0,178-2,556$ \\
\hline Mellitus & & & & & \\
\hline $\begin{array}{l}\text { Hipertensión } \\
\text { arterial }\end{array}$ & $4(13,8)$ & $26(86,7)$ & 0,036 & 0,367 & $0,133-1,015$ \\
\hline \multicolumn{6}{|c|}{ Otros medicamentos. Inductores enzimáticos } \\
\hline Omeprazol & $3(20)$ & $12(80)$ & 0,740 & 0,738 & $0,243-2,249$ \\
\hline Lovastastina & $10(29,4)$ & $24(70,6)$ & 0,428 & 1,422 & $0,588-3,436$ \\
\hline \multicolumn{6}{|l|}{ Indicación Warfarina } \\
\hline $\begin{array}{l}\text { Trombosis } \\
\text { venosas } \\
\text { profunda }\end{array}$ & $3(37,5)$ & $5(62,5)$ & 0,400 & 1,587 & $0,576-4,369$ \\
\hline $\begin{array}{l}\text { Fibrilación } \\
\text { auricular }\end{array}$ & $6(23,1)$ & $20(76,9)$ & 0,723 & 0,854 & $0,354-2,057$ \\
\hline $\begin{array}{l}\text { Trombosis } \\
\text { pulmonar }\end{array}$ & $1(16,7)$ & $5(83,3)$ & 1,000 & 0,633 & $0,100-3,992$ \\
\hline Prótesis valvular & $6(26,1)$ & $17(73,9)$ & 1,000 & 1,043 & $0,436-2,497$ \\
\hline \multirow[t]{2}{*}{$\begin{array}{l}\text { Variables } \\
\text { cuantitativas }\end{array}$} & INR $\geq 0,5$ & INR $<0,5$ & Valor $\mathrm{p}^{\mathrm{a}, \mathrm{b}}$ & $\mathrm{RR}$ & IC del RR \\
\hline & n (\%) & $\mathrm{n}(\%)$ & & & \\
\hline Edad $\geq 70$ años & $15(46,9)$ & $21(67,7)$ & 0,094 & 3,023 & $0,760-12,020$ \\
\hline$I M C \geq 25$ & $11(25,6)$ & $32(74,4)$ & 1,000 & 1,023 & $0,410-2,553$ \\
\hline \multicolumn{6}{|c|}{ Dosis warfarina (mg/semana) } \\
\hline$\leq 20$ & $1(12,5)$ & $7(87,5)$ & 0,667 & 0,458 & $0,070-3,014$ \\
\hline $20-30$ & $4(25,0)$ & $12(75,0)$ & 1,000 & 0,979 & $0,368-2,607$ \\
\hline $30-40$ & $5(23,8)$ & $16(76,2)$ & 1,000 & 0,909 & $0,363-2,277$ \\
\hline $40-50$ & $3(20,0)$ & $12(80,0)$ & 0,582 & 0,738 & $0,243-2,249$ \\
\hline$\geq 50$ & $4(44,4)$ & $5(55,6)$ & 0,156 & 2,000 & $0,826-4,844$ \\
\hline \multicolumn{6}{|l|}{ INR Mes - 1} \\
\hline$<2,5$ & $6(25,0)$ & $18(75,0)$ & 0,955 & 0,975 & $0,406-2,339$ \\
\hline $2,5-3,0$ & $6(27,3)$ & $16(72,7)$ & 0,802 & 1,118 & $0,469-2,668$ \\
\hline$>3,0$ & $4(21,1)$ & $15(78,9)$ & 0,757 & 0,772 & $0,285-2,089$ \\
\hline \multicolumn{6}{|l|}{ INR Mes 0} \\
\hline$<2,5$ & $7(25,9)$ & $20(74,1)$ & 0,933 & 1,037 & $0,442-2,432$ \\
\hline $2,5-3,0$ & $7(36,8)$ & $12(63,2)$ & 0,170 & 1,801 & $0,787-4,124$ \\
\hline$>3,0$ & $2(11,8)$ & $15(88,2)$ & 0,131 & 0,387 & $0,980-1,526$ \\
\hline
\end{tabular}

a Test chi-cuadrado de Pearson.

b Prueba Fisher's Exacto.

no encontró que estas variables estuvieran asociadas con el aumento del INR $\geq 0,5$ unidades. La indicación de la warfarina en PV fue la única variable que tuvo una significancia muy cercana al límite de aceptación (valor $p=0,052$ ).
En el presente estudio, se evidenció que los médicos tratantes de la cohorte expuesta al FR, luego de notar que los pacientes tuvieron un incremento del INR al final del seguimiento, tomaron decisiones clínicas como la 
Tabla 3 Descripción del análisis estratificado para todas las variables

\begin{tabular}{|c|c|c|c|c|}
\hline & INR $\geq 0,5$ & INR $<0,5$ & Valor $\mathrm{p}^{\mathrm{a}, \mathrm{b}}$ & Valor $\mathrm{p}^{\mathrm{c}}$ \\
\hline & $\mathrm{n}(\%)$ & n (\%) & & \\
\hline \multicolumn{5}{|l|}{ Otras concomitantes } \\
\hline$\geq 3$ enfermedades & $2(20)$ & $8(80)$ & 1,000 & 0,063 \\
\hline$<3$ enfermedades & $7(63,6)$ & $4(36,4)$ & 0,011 & \\
\hline Con dislipidemia & $2(22,79$ & $7(77,8)$ & 0,095 & 0,058 \\
\hline Sin dislipidemia & $7(58,3)$ & $5(41,7)$ & 0,002 & \\
\hline \multicolumn{5}{|l|}{ Polimedicación } \\
\hline$\geq 6$ medicamentos & $3(37,59$ & $5(62,59$ & 0,346 & 0,054 \\
\hline$<5$ medicamentos & $6(46,2)$ & $7(53,8)$ & 0,043 & \\
\hline \multicolumn{5}{|c|}{ Otros medicamentos enzimáticos } \\
\hline Con lovastastina & $3(50)$ & $3(50)$ & 0,328 & 0,016 \\
\hline Sin lovastastina & $6(40)$ & $9(60)$ & 0,017 & \\
\hline \multicolumn{5}{|l|}{ Indicación warfarina } \\
\hline Con Fibrilación Auricular & $2(25)$ & $6(75)$ & 1,000 & 0,057 \\
\hline Sin Fibrilación auricular & $7(53,8)$ & $6(46,2)$ & 0,017 & \\
\hline Con prótesis valvular & $4(66,7)$ & $2(33,3)$ & 0,021 & 0,052 \\
\hline Sin Prótesis valvular & $5(33,3)$ & $10(66,7)$ & 0,457 & \\
\hline \multicolumn{5}{|l|}{ Dosis warfarina (mg/semana) } \\
\hline$\geq 50$ & $1(50)$ & $1(50)$ & 1,000 & 0,036 \\
\hline$<50$ & $8(42,1)$ & $11(57,9)$ & 0,016 & \\
\hline \multicolumn{5}{|l|}{ INR.Mes 0} \\
\hline$\geq 3$ & $2(50)$ & $2(50)$ & 0,044 & 0,08 \\
\hline$<3$ & $7(41,2)$ & $10(58,8)$ & 0,225 & \\
\hline
\end{tabular}

descontinuación de la warfarina en 4 pacientes (19\%). Se encontró que solo un médico tratante reportó en la historia clínica que el incremento del INR pudo estar relacionado con el uso concomitante de acetaminofén en el mes anterior de la consulta de seguimiento respectiva. Generalmente, se considera que la prescripción del acetaminofén con la warfarina puede afectar los resultados de las dosis de mantenimiento de la warfarina ${ }^{30}$. En este sentido, aunque la interacción medicamentosa entre la warfarina y el acetaminofén ha sido publicada desde hace más de 50 de $\operatorname{años}^{31}$, aún existe poca sensibilización y conocimiento en la identificación y prevención de la misma, y su asociación con el riesgo del aumento del INR y eventos hemorrágicos. Esta situación ha sido señalada en algunos reportes de casos, en los cuales pacientes con terapia anticoagulante estable con la warfarina cuando utilizaban el acetaminofén presentaban un incremento clínicamente representativo del INR, llevando en uno de los casos a la manifestación de un hematoma retroperitoneal ${ }^{32,33}$, lo que ha permitido establecer la causalidad del acetaminofén en el incremento del INR.

Otros estudios han demostrado que el riesgo de hemorragia está estrechamente relacionado con la intensidad de la anticoagulación, siendo significativamente, mayor cuando el INR es igual o mayor a tres unidades y aumenta luego exponencialmente ${ }^{34}$. Otros investigadores como Mahe, et

Tabla 4 Regresión logística multivariable de los factores que presumiblemente pudieran aumentar el INR $\geq 0,5$ unidades

\begin{tabular}{|c|c|c|c|c|c|c|c|}
\hline \multirow[t]{2}{*}{ Variable } & \multirow[t]{2}{*}{ B } & \multirow[t]{2}{*}{ E.E. } & \multirow[t]{2}{*}{ Wald } & \multirow[t]{2}{*}{ Sig. } & \multirow[t]{2}{*}{$\operatorname{Exp}(B)$} & \multicolumn{2}{|c|}{ (IC 95\%) } \\
\hline & & & & & & Inferior & Superior \\
\hline Dosis warfarina $\geq 50 \mathrm{mg} / \mathrm{sem}$ & 2,574 & 1,128 & 5,210 & 0,022 & 13,121 & 1,439 & 119,653 \\
\hline Edad $\geq 70$ & 1,775 & 0,878 & 4,091 & 0,043 & 5,901 & 1,057 & 32,961 \\
\hline Expuestos & 1,295 & 0,717 & 3,261 & 0,071 & 3,650 & 0,895 & 14,877 \\
\hline INR $\geq 3$ & $-0,626$ & 0,923 & 0,460 & 0,498 & 0,535 & 0,088 & 3,262 \\
\hline HTA & $-1,169$ & 0,737 & 2,517 & 0,113 & 0,311 & 0,073 & 1,317 \\
\hline Constante & $-1,363$ & 1,542 & 0,781 & 0,377 & 0,256 & & \\
\hline
\end{tabular}


$\mathrm{al}^{12}$, consideran que este riesgo tiene mayor relevancia clínica en los pacientes con medición del INR igual o mayor a 3,5 unidades después de iniciar el uso del acetaminofén, siendo aún mayor a valores de INR igual o mayor a cuatro unidades. Relacionado con estos hallazgos, en el presente estudio no se presentaron consultas por urgencia u hospitalizaciones relacionadas con eventos hemorrágicos después de la exposición al acetaminofén, no obstante, 6 pacientes $(28,5 \%)$, tuvieron un INR final igual o mayor a 3,5 unidades.

Respecto a la relevancia clínica, acorde con la metodología propuesta por Amariles, et al., la interacción entre la warfarina y el acetaminofén sería de un nivel 2 (riesgo alto). Este nivel se explica por el cruce de la gravedad (moderada) con una probabilidad de aparición (definida). La valoración de la gravedad de la interacción como moderada se debe a que causa una modificación (cambio o interrupción), en la farmacoterapia del paciente. Es importante destacar que, a pesar que la interacción puede causar daño o lesión al paciente por el aumento del INR, en este estudio, ni en los publicados a la fecha se han documentado hemorragias que pusieran en riesgo la vida del paciente, excepto en un reporte en el cual un paciente presentó una hemorragia retroperitoneal. Por su parte, la valoración de probabilidad de aparición de la interacción como definida, se debe a que la misma ha sido demostrada mediante $\mathrm{ECC}^{32}$.

Se recomienda que el uso del acetaminofén en la terapia anticoagulante con la warfarina debe hacerse bajo una estrecha monitorización para evitar incrementos en el INR y disminuir el riesgo de sangrado, incluso a dosis menores de 2 gramos/día como se demostró en este estudio, aunque la mayor evidencia publicada establece que esta interacción se presenta a altas dosis del acetaminofén (mayor a 2 gramos/día) o por su uso prolongado, en este sentido, se recomienda utilizar una dosis menor a 2 gramos al día por 3-4 días como máximo.

\section{Limitaciones}

La información se tomó directamente de las historias clínicas, lo que pudo generar sesgos de información. No fue posible obtener más de una medición del INR por paciente durante el mes 0 y el mes 1 de seguimiento, luego de la exposición al FR, debido a que estos datos fueron recopilados de las historias clínicas de un programa de pacientes anticoagulados con la warfarina, en el cual se tiene establecido realizar mediciones de monitorización del INR cada 30 días como mínimo. Por último, las variaciones previas a los 28,7 días del INR no fueron determinadas, lo que pudo afectar la identificación de evento final, tal como se ha establecido por Mahe, et al. y Parra, et al ${ }^{12,15}$, estudios en los que se han evidenciado aumentos significativos del INR, incluso igual o mayor a 0,6 unidades a los 7 días de exposición al FR.

\section{Conclusión}

Los resultados de este estudio respaldan que, en pacientes anticoagulados con warfarina, la utilización del acetaminofén genera un aumento del INR igual o mayor de 0,5 unidades, asociado a un mayor efecto anticoagulante $y$, por tanto, a un mayor riesgo de sangrado.

\section{Responsabilidades éticas}

Protección de personas y animales. Los autores declaran que para esta investigación no se han realizado experimentos en seres humanos ni en animales.

Confidencialidad de los datos. Los autores declaran que en este artículo no aparecen datos de pacientes.

Derecho a la privacidad y consentimiento informado. Los autores declaran que en este artículo no aparecen datos de pacientes.

\section{Financiación}

El grupo Promoción y Prevención Farmacéutica recibió financiación de la convocatoria de sostenibilidad 2014-2015 del Comité para el Desarrollo de la Investigación de la Universidad de Antioquia.

\section{Conflicto de intereses}

Los autores declaran no tener ningún conflicto de intereses.

\section{Bibliografía}

1. Meckley LM, Gudgeon JM, Anderson JL, Williams MS, Veenstra DL. A policy model to evaluate the benefits, risks and costs of warfarin pharmacogenomic testing. Pharmacoeconomics. 2010;28:61-74.

2. Fihn SD, Callahan CM, Martin DC, McDonell MB, Henikoff JG, White $\mathrm{RH}$. The risk for and severity of bleeding complications in elderly patients treated with warfarin. The National Consortium of Anticoagulation Clinics. Ann. Intern. Med. 1996;124:970-9.

3. Hylek EM, Heiman H, Skates SJ, Sheehan MA, Singer DE. Acetaminophen and other risk factors for excessive warfarin anticoagulation. JAMA. 1998;279:657-62.

4. Joffe HV, Xu R, Johnson FB, Longtine J, Kucher N, Goldhaber SZ. Warfarin dosing and cytochrome P450 2C9 polymorphisms. Thromb. Haemost. 2004;91:1123-8.

5. Hirsh J, Dalen J, Anderson DR, Poller L, Bussey H, Ansell J, et al. Oral anticoagulants: mechanism of action, clinical effectiveness, and optimal therapeutic range. Chest. 2001;119:8S-21S.

6. Rikala M, Hauta-Aho M, Helin-Salmivaara A, Lassila R, Korhonen MJ, Huupponen R. Co-Prescribing of Potentially Interacting Drugs during Warfarin Therapy - A Population-Based Register Study. Basic Clin. Pharmacol. Toxicol. 2014;117:126-32.

7. Jacobs LG. Warfarin pharmacology, clinical management, and evaluation of hemorrhagic risk for the elderly. Cardiol. Clin. 2008;26:157-67.

8. Rosand J, Eckman MH, Knudsen KA, Singer DE, Greenberg SM. The effect of warfarin and intensity of anticoagulation on outcome of intracerebral hemorrhage. Arch. Intern. Med. 2004;164:80-4.

9. Fric-Shamji EC, Shamji MF, Cole J, Benoit BG. Modifiable risk factors for intracerebral hemorrhage: study of anticoagulated patients. Can. Fam. Physician. 2008;54:1138-9, 139 e1-4.

10. Aguilar MI, Hart RG, Kase CS, Freeman WD, Hoeben BJ, García RC, et al. Treatment of warfarin-associated intracerebral hemorrhage: literature review and expert opinion. Mayo Clin. Proc. 2007;82:82-92.

11. Francisco JYR. Wafarina: uso contemporaneo. Rev. Med. 2009;17:107-15. 
12. Mahé I, Bertrand N, Drouet L, Bal Dit Sollier C, Simoneau G, Mazoyer $\mathrm{E}$, et al. Interaction between paracetamol and warfarin in patients: a double-blind, placebo-controlled, randomized study. Haematológica. 2006;91:1621-7.

13. Mahé I, Bertrand N, Drouet L, Simoneau G, Mazoyer E, Bal Dit Sollier C, et al. Paracetamol: a haemorrhagic risk factor in patients on warfarin. Br. J. Clin. Pharmacol. 2005;59:371-4.

14. Kwan D, Bartle WR, Walker SE. The effects of acetaminophen on pharmacokinetics and pharmacodynamics of warfarin. J. Clin. Pharmacol. 1999;39:68-75.

15. Parra D, Beckey NP, Stevens GR. The effect of acetaminophen on the international normalized ratio in patients stabilized on warfarin therapy. Pharmacotherapy. 2007;27:675-83.

16. Schulman S. Clinical practice. Care of patients receiving longterm anticoagulant therapy. N. Engl. J. Med. 2003;349:675-83.

17. Thijssen HH, Soute BA, Vervoort LM, Claessens JG. Paracetamol (acetaminophen) warfarin interaction: NAPQI, the toxic metabolite of paracetamol, is an inhibitor of enzymes in the vitamin K cycle. Thromb. Haemost. 2004;92:797-802.

18. Lassen JF, Kjeldsen J, Antonsen S, Hyltoft Petersen P, Brandslund I. Interpretation of serial measurements of international normalized ratio for prothrombin times in monitoring oral anticoagulant therapy. Clin. Chem. 1995;41:1171-6.

19. Ceballos M, Holguín H, González C, Amariles P. Warfarin and acetaminophen interaction Assessment to establish its clinical relevance. Acta Medica Colomb. 2013;38:22-5.

20. Amariles P, Giraldo NA, Faus MJ. Clinical relevance of drug interactions. Med. Clin. (Barc). 2007;129:27-35.

21. Whyte IM, Buckley NA, Reith DM, Goodhew I, Seldon M, Dawson $\mathrm{AH}$. Acetaminophen causes an increased International Normalized Ratio by reducing functional factor VII. Ther. Drug Monit. 2000;22:742-8.

22. Choi KH, Kim AJ, Son IJ, Kim KH, Kim KB, Ahn H, et al. Risk factors of drug interaction between warfarin and nonsteroidal anti-inflammatory drugs in practical setting. J. Korean Med. Sci. 2010;25:337-41.

23. Builes CE, Arango A, Cano WM. Frequency of bleeding in patients with cardiovascular disease on anticoagulant therapy with generic warfarin vs. Coumadin. Acta Medica Colomb. 2010;35: $175-8$.

24. Lee HL, Kan CD, Yang YJ. Efficacy and tolerability of the switch from a branded to a generic warfarin sodium product: an observer-blinded, randomized, crossover study. Clin. Ther. 2005;27:309-19.

25. Swenson CN, Fundak G. Observational cohort study of switching warfarin sodium products in a managed care organization. Am. J. Health. Syst. Pharm. 2000;57:452-5.

26. Milligan PE, Banet GA, Waterman AD, Gatchel SK, Gage BF. Substitution of generic warfarin for Coumadin in an HMO setting. Ann. Pharmacother. 2002;36:764-8.

27. Resolución 8430 de 1993. at <http://www.minsalud.gov.co/ Normatividad/RESOLUCION 8430 DE 1993.pdf>.

28. Kotirum S, Chaiyakunapruk N, Jampachaisri K, Wattanasombat $\mathrm{S}$, Rojnuckarin P. Utilization review of concomitant use of potentially interacting drugs in Thai patients using warfarin therapy. Pharmacoepidemiol. Drug Saf. 2007; 16:216-22.

29. Anderson BJ. Paracetamol (Acetaminophen): mechanisms of action. Paediatr. Anaesth. 2008;18:915-21.

30. Shalansky S, Lynd L, Richardson K, Ingaszewski A, Kerr C. Risk of warfarin-related bleeding events and supratherapeutic international normalized ratios associated with complementary and alternative medicine: a longitudinal analysis. Pharmacotherapy. 2007;27:1237-47.

31. Antlitz AM, Mead JA, Tolentino MA. Potentiation of oral anticoagulant therapy by acetaminophen. Curr. Ther. Res. Clin. Exp. 1968; 10:501-7.

32. Andrews LB, Bridgeman MB, Dalal KS, Abazia D, Lau C, Goldsmith DF, et al. Implementation of a pharmacist-driven pain management consultation service for hospitalised adults with a history of substance abuse. Int. J. Clin. Pract. 2013;67:1342-9.

33. Gebauer MG, Nyfort-Hansen K, Henschke PJ, Gallus AS. Warfarin and acetaminophen interaction. Pharmacotherapy. 2003;23:109-12.

34. Hylek EM, Singer DE. Risk factors for intracranial hemorrhage in outpatients taking warfarin. Ann. Intern. Med. 1994;120:897-902. 